\title{
Sampling Reference Points on the Pareto Fronts of Multi-Objective Optimization Problems
}

\author{
Ye Tian ${ }^{1}$, Xiaoshu Xiang ${ }^{1}$, Xingyi Zhang ${ }^{1}$, Ran Cheng ${ }^{2}$, and Yaochu Jin ${ }^{3}$ \\ ${ }^{1}$ Institute of Bio-inspired Intelligence and Mining Knowledge, School of Computer Science and Technology, \\ Anhui University, Hefei 230039, China (Corresponding author: Xingyi Zhang) \\ ${ }^{2}$ School of Computer Science, University of Birmingham, Birmingham, B15 2TT, United Kingdom \\ ${ }^{3}$ Department of Computer Science, University of Surrey, Guildford, Surrey, GU2 7XH, United Kingdom \\ Email: \{field910921@gmail.com; xxs1394@163.com; xyzhanghust@gmail.com; ranchengcn@gmail.com; yaochu.jin@surrey.ac.uk\}
}

\begin{abstract}
The effectiveness of evolutionary algorithms have been verified on multi-objective optimization, and a large number of multi-objective evolutionary algorithms have been proposed during the last two decades. To quantitatively compare the performance of different algorithms, a set of uniformly distributed reference points sampled on the Pareto fronts of benchmark problems are needed in the calculation of most performance metrics. However, not much work has been done to investigate the method for sampling reference points on Pareto fronts, even though it is not an easy task for many Pareto fronts with irregular shapes. More recently, an evolutionary multi-objective optimization platform was proposed by us, called PlatEMO, which can automatically generate reference points on each Pareto front and use them to calculate the performance metric values. In this paper, we report the reference point sampling methods used in PlatEMO for different types of Pareto fronts. Experimental results show that the reference points generated by the proposed sampling methods can evaluate the performance of algorithms more accurately than randomly sampled reference points.
\end{abstract}

\section{INTRODUCTION}

Since the vector evaluated genetic algorithm (VEGA) was proposed in 1985 [1], a large number of evolutionary algorithms have been proposed and demonstrated high effectiveness in solving multi-objective optimization problems (MOPs), which are collectively known as multi-objective evolutionary algorithms (MOEAs) [2], [3]. In order to compare the performance of existing MOEAs, some multi-objective test suites with various types of Pareto sets, Pareto fronts, and landscapes have been proposed. For example, ZDT [4] is one of the first test suites which contains six MOPs with two objectives; DTLZ [5] and WFG [6] are the two most popular test suites, which are scalable with respect to both decision variables and objective; UF [7] contains ten bi- and three-objective MOPs with complicated linkages between decision variables, which pose tough challenges to MOEAs to obtain a set of wellconverged solutions; LSMOP [8] is the first test suite designed for large-scale multi-objective optimization, and $\mathrm{MaF}$ [9] is a recently proposed test suite for many-objective optimization.

Since each solution for an MOP has multiple objective values, it is difficult to evaluate the performance of MOEAs by directly comparing the objective values of the solutions. Therefore, some performance metrics have been proposed to quantitatively evaluate the outcomes of MOEAs, such as generational distance (GD) [10], inverted generational distance (IGD) [11], and hypervolume (HV) [12]. It should be noted that, a set of uniformly distributed reference points sampled on the Pareto front $(\mathrm{PF})$ is needed as the ground-truth for the calculation of some performance metrics. For instances, the IGD is calculated by

$$
I G D(P, R)=\frac{\sum_{\mathbf{r} \in R} \min _{\mathbf{p} \in P}\|\mathbf{p}-\mathbf{r}\|}{|R|},
$$

where $P$ is the objective values of a solution set, $R$ is the reference point set, and $\|\cdot\|$ is the $L_{2}$-norm. In short, IGD calculates an average minimum distance from each point in $R$ to those in $P$, where a smaller IGD value indicates a better convergence and diversity of $P$.

However, it is not an easy task to sample a set of uniformly distributed reference points on various PFs, and only few work has been done to investigate the reference point sampling method [13], [14]. To address this issue, this paper systematically presents the reference point sampling methods used in PlatEMO [15], which is an evolutionary multi-objective optimization platform recently proposed by us. PlatEMO can automatically generate a set of uniformly distributed reference points on each PF, and use them to calculate the performance metric values of the solutions obtained by MOEAs.

The rest of this paper is organized as follows. Section II reviews three methods for sampling reference points on unit simplex, which is the prerequisite for sampling reference points on most PFs. Section III introduces the reference point sampling methods for several types of PFs. Section IV presents the experimental results of five popular MOEAs on five MOPs, to verify the effectiveness of the proposed reference point sampling methods in performance metric calculation. Conclusions are drawn in Section V.

\section{Sampling Reference Points on Unit Simplex}

The uniformly distributed reference points on most PFs can be obtained by transforming the points uniformly sampled on unit simplex. Among many others [16], [17], three representative methods for sampling reference points on unit simplex are reviewed in the following. 


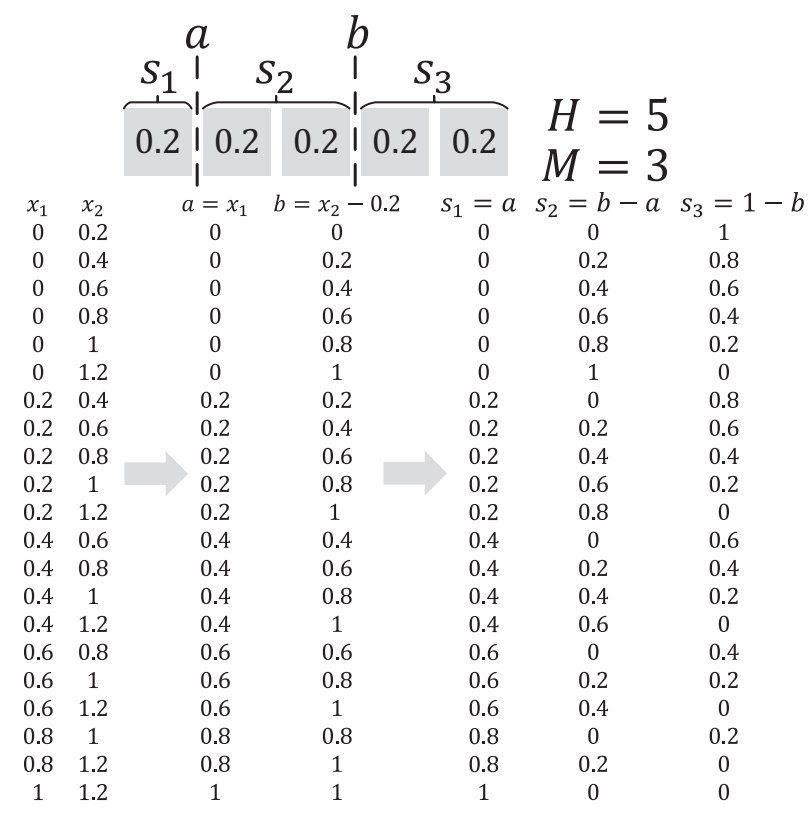

Fig. 1. Example of generating reference points by Das and Dennis's method.

\section{A. Das and Dennis's Method}

The Das and Dennis's method [18] is the most popular systematic approach for sampling uniformly distributed reference points on unit simplex, which is commonly employed by decomposition based MOEAs [19]. A reference point on $M$-dimensional unit simplex generated by Das and Dennis's method can be defined by $\mathbf{s}=\left(s_{1}, s_{2}, \ldots, s_{M}\right)$, where

$$
s_{j} \in\left\{\frac{0}{H}, \frac{1}{H}, \ldots, \frac{H}{H}\right\}, \sum_{j=1}^{M} s_{j}=1
$$

and $H$ is the number of divisions in each objective.

Fig. 1 illustrates the procedure for generating all the points satisfying (2) with $M=3$ and $H=5$. Specifically, we can find all the combinations of $a$ and $b$ that satisfy $a, b \in$ $\{0,0.2, \ldots, 1\}$ and $a \leq b$, then let $s_{1}=a-0, s_{2}=b-a$, and $s_{3}=1-b$. Therefore, the above issue is equivalent to finding all the 2-multicombinations of $\{0,0.2, \ldots, 1\}$, which can be further converted to finding all the 2-combinations of $\{0,0.2, \ldots, 1.2\}$. To summarize, the procedure of Das and Dennis's method is:

1) Let $X$ be all the $(M-1)$-combinations of $\left\{\frac{0}{H}, \frac{1}{H}\right.$, $\left.\ldots, \frac{H+M-2}{H}\right\}$

2) For each $x_{i j} \in X$ (i.e., the $j$-th element of the $i$-th combination in $X), x_{i j}=x_{i j}-\frac{j-1}{H}$;

3) Let $S$ be the reference point set, for each $s_{i j} \in S$ and $x_{i j} \in X$,

$$
\left\{\begin{array}{ll}
s_{i j}=x_{i j}-0, & j=1 \\
s_{i j}=x_{i j}-x_{i(j-1)}, & 1<j<M \\
s_{i j}=1-x_{i(j-1)}, & j=M
\end{array} .\right.
$$

Fig. 2 plots the reference points generated by Das and Dennis's method with $M=3, H=13$ and $M=10, H=3$. From Step 1), it is known that the number of reference points generated by Das and Dennis's method is $C_{H+M-1}^{M-1}$,

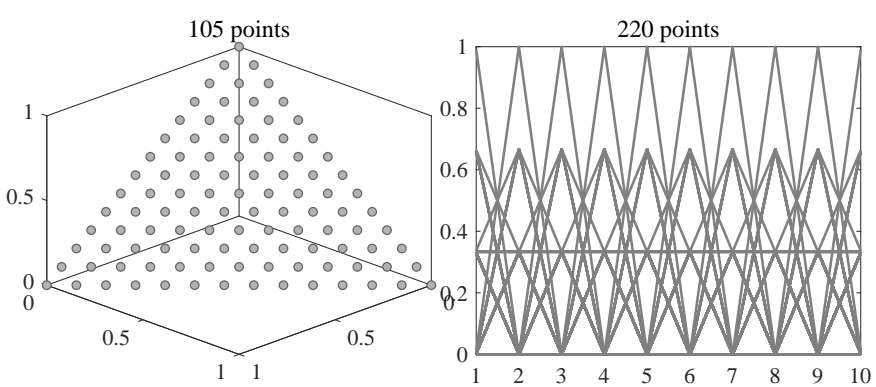

Fig. 2. The reference points sampled by Das and Dennis's method on 3and 10-objective unit simplexes.

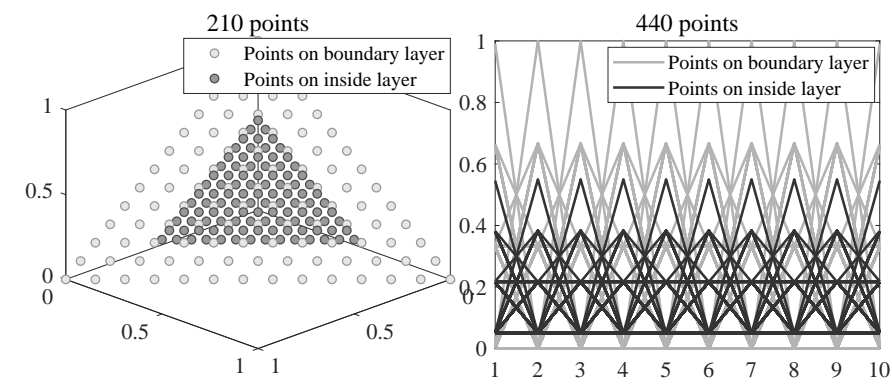

Fig. 3. The reference points sampled by Deb and Jain's method on 3- and 10-objective unit simplexes.

hence the numbers of reference points shown in Fig. 2 are $C_{13+3-1}^{3-1}=105$ and $C_{3+10-1}^{10-1}=220$, respectively.

\section{B. Deb and Jain's Method}

As reported by Deb and Jain [20], no intermediate point will be generated by Das and Dennis's method as long as $H<M$. Therefore, at least $C_{10+10-1}^{10-1}=92378$ points on 10-objective unit simplex are required to have at least one intermediate point. To avoid such a situation, they suggested to use two layers of reference points, which are generated as follows:

1) Generate $S_{1}$ by Das and Dennis's method as the point set on boundary layer;

2) Let $S_{2}$ be the point set on inside layer, for each $s_{i j}^{\prime} \in S_{2}$ and $s_{i j} \in S_{1}$,

$$
s_{i j}^{\prime}=\frac{1}{2} s_{i j}+\frac{1}{2 M}
$$

3) The reference point set $S=S_{1} \cup S_{2}$.

Fig. 3 depicts the reference points generated by Deb and Jain's method with $M=3, H=13$ and $M=10, H=$ 3 , where the numbers of reference points are 210 and 440 , respectively. It can be seen from the figure that the reference points on boundary layer are the same to those generated by Das and Dennis's method, while the reference points on inside layer are shrunk to the center of the simplexes. So there can exist intermediate point even if $H<M$. In practice, the Das and Dennis's method is used when $M \leq 5$, and the Deb and Jain's method is used in other cases.

\section{Mixture Uniform Design}

A drawback of the above two sampling methods is that the number of reference points is restricted by the parameters $M$ and $H$. By contrast, the mixture uniform design [21] provides a 

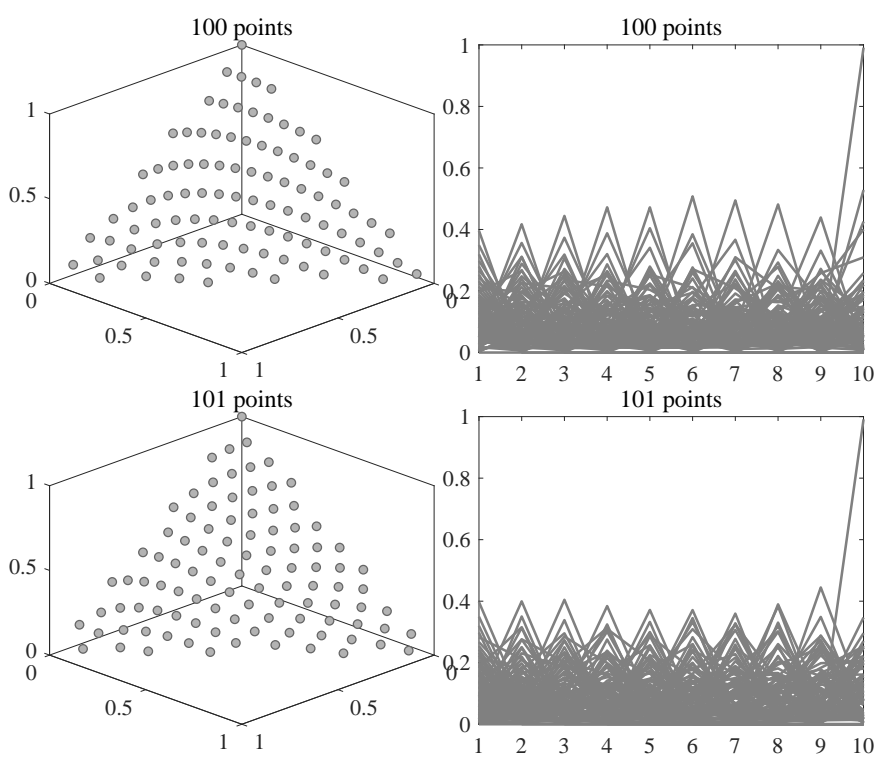

Fig. 4. The reference points sampled by mixture uniform design on 3- and 10-objective unit simplexes.

more flexible sampling method, where the number of reference points can be an arbitrary number. In short, it generates $N$ points uniformly distributed in an $(M-1)$-dimensional hypercube, then transforms them to the $M$-dimensional unit simplex. Specifically, the procedure of mixture uniform design consists of the following four steps:

1) Let $W_{1}$ be a row vector of all the positive integers that are coprime to and smaller than $N$; let $W_{2}=$ $(1,2, \ldots, N)$;

2) Let $W=\bmod \left(W_{2}^{T} W_{1}-1, N\right)+1$;

3) Let $X$ be a matrix consisting of $M-1$ columns from $W$, find the $X$ having the largest $C D_{2}$ value;

4) Let $S$ be the reference point set, for each $s_{i j} \in S$ and $x_{i j} \in X$,

$$
\begin{cases}s_{i j}=\frac{1}{N}\left[1-\left(x_{i j}\right)^{\frac{1}{M-j}}\right] \prod_{k=1}^{j-1}\left(x_{i k}\right)^{\frac{1}{M-k}}, & 1 \leq j<M \\ s_{i j}=\frac{1}{N} \prod_{k=1}^{j-1}\left(x_{i k}\right)^{\frac{1}{M-k}}, & j=M\end{cases}
$$

In Step 3), the $C D_{2}$ (i.e., centered $L_{2}$-discrepancy) is used to measure the diversity of a point set $X$ with $N$ points filling a hypercube, which can be calculated by

$$
\begin{aligned}
C D_{2}(X)= & {\left[\left(\frac{13}{12}\right)^{M-1}-\frac{2^{2-M}}{N} \sum_{i=1}^{N} \prod_{j=1}^{M-1}\left(2+\left|x_{i j}-0.5\right|-\right.\right.} \\
& \left.\left|x_{i j}-0.5\right|^{2}\right)+\frac{1}{N^{2}} \sum_{i, k=1}^{N} \prod_{j=1}^{M-1}\left(1+0.5 \mid x_{i j}-\right. \\
& \left.\left.0.5|+0.5| x_{k j}-0.5|-0.5| x_{i j}-x_{k j} \mid\right)\right]^{\frac{1}{2}}
\end{aligned}
$$

Assume that the matrix $W$ contains $K$ columns, the calculation of $C D_{2}$ should be performed for $C_{K}^{M-1}$ times, hence the computational complexity of mixture uniform design is obviously larger than those of Das and Dennis's method and Deb and Jain's method.

Fig. 4 presents 100 and 101 reference points generated by mixture uniform design with $M=3$ and $M=10$. It is

\footnotetext{
${ }^{1}$ Step 1) - Step 3) is called the good lattice point method, other methods such as the Latin hypercube sampling can also be adopted for generating $X$.
}

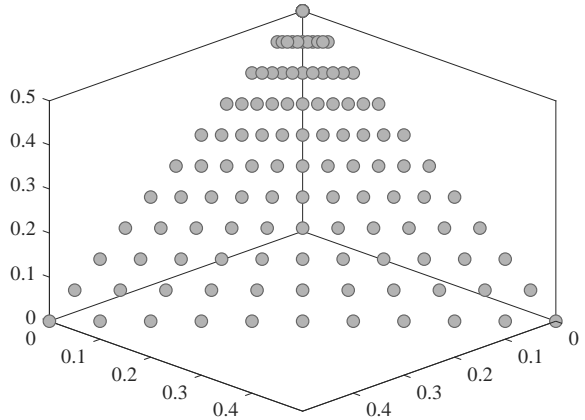

Fig. 5. Reference points for 3-objective DTLZ1, which are generated by uniformly sampling decision variables.

obvious from the figure that the number of reference points can be an arbitrary number; however, the obtained reference points distribute less uniformly than those generated by Das and Dennis's method, and many extreme points are missed.

\section{Sampling Reference Points on PAREto Fronts}

In this section, we give the reference point sampling methods for several types of PFs. The Das and Dennis's method is adopted to generate the uniformly distributed reference points on unit simplex, unless otherwise specified.

\section{A. Sampling Reference Points on Linear PF (DTLZ1)}

The PF of $M$-objective DTLZ1 [5] is defined by

$$
\left\{\begin{array}{l}
f_{1}=0.5 x_{1} x_{2} \ldots x_{M-1} \\
f_{2}=0.5 x_{1} x_{2} \ldots\left(1-x_{M-1}\right) \\
\ldots \\
f_{M}=0.5\left(1-x_{1}\right)
\end{array},\right.
$$

where $0 \leq x_{j} \leq 1$. As shown in Fig. 5, if we uniformly generate $x_{j}$ and calculate the reference points by (7), the obtained reference points will distribute nonuniformly. Therefore, it needs to sample the reference points in objective space directly. In fact, the PF of $M$-objective DTLZ1 [5] can be written as

$$
f_{1}+f_{2}+\ldots+f_{M}=0.5,
$$

where $0 \leq f_{j} \leq 0.5$. So the reference points can be obtained by halving the objective values of the points on unit simplex, i.e.,

1) Uniformly sample a set of points $S$ on unit simplex;

2) Let $R$ be the reference point set, for each $r_{i j} \in R$ and $s_{i j} \in S$,

$$
r_{i j}=0.5 s_{i j}
$$

Fig. 6 plots 120 reference points sampled on 3-objective DTLZ1.

B. Sampling Reference Points on Concave and Convex PFs (DTLZ2 and Its Variants)

This subsection introduces the reference point sampling methods for DTLZ2 [5] and three of its variants, namely, convex DTLZ2 [20], C2-DTLZ2 [22], and DTLZ2BZ [23]. 


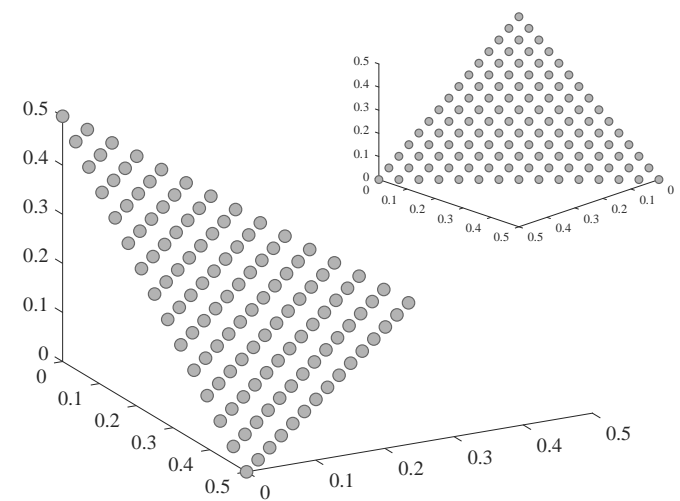

Fig. 6. 120 reference points sampled on 3-objective DTLZ1.

The reference points sampled on the PFs of these four MOPs are plotted in Fig. 7.

The PF of $M$-objective DTLZ2 can be written as

$$
f_{1}^{2}+f_{2}^{2}+\ldots+f_{M}^{2}=1,
$$

where $0 \leq f_{j} \leq 1$. The reference points for DTLZ2 can be obtained by mapping the points from unit simplex to unit sphere, i.e., calculating the intersection of the line connecting each point and the origin on unit sphere:

1) Uniformly sample a set of points $S$ on unit simplex;

2) Let $R$ be the reference point set, for each $r_{i j} \in R$ and $s_{i j} \in S$,

$$
r_{i j}=\frac{s_{i j}}{t_{i}},
$$

where $t_{i}$ can be obtained by solving

$$
\left(\frac{s_{i 1}}{t_{i}}\right)^{2}+\left(\frac{s_{i 2}}{t_{i}}\right)^{2}+\ldots+\left(\frac{s_{i M}}{t_{i}}\right)^{2}=1
$$

i.e.,

$$
t_{i}=\sqrt{\sum_{k=1}^{M} s_{i k}^{2}} .
$$

The PF of $M$-objective convex DTLZ2 is

$$
f_{1}^{0.5}+f_{2}^{0.5}+\ldots+f_{M-1}^{0.5}+f_{M}=1,
$$

where $0 \leq f_{j} \leq 1$. Similar to DTLZ2, the procedure of sampling reference points on the PF of convex DTLZ2 is

1) Uniformly sample a set of points $S$ on unit simplex;

2) Let $R$ be the reference point set, for each $r_{i j} \in R$ and $s_{i j} \in S$,

$$
\begin{aligned}
& r_{i j}=\frac{s_{i j}}{t_{i}} \\
& t_{i}=\frac{\delta_{i}+2 s_{i M}+\sqrt{\delta_{i}^{2}+4 \delta_{i} s_{i M}}}{2} . \\
& \delta_{i}=\left(\sum_{k=1}^{M-1} \sqrt{s_{i k}}\right)^{2}
\end{aligned}
$$

The PF of $M$-objective C2-DTLZ2 is the same to DTLZ2, except that a constraint is introduced

$$
\begin{gathered}
\min \left\{\min _{j=1}^{M}\left[\left(f_{j}-1\right)^{2}+\sum_{k=1, k \neq j}^{M} f_{k}^{2}-a^{2}\right],\right. \\
\left.\left[\sum_{j=1}^{M}\left(f_{j}-1 / \sqrt{M}\right)^{2}-a^{2}\right]\right\} \leq 0,
\end{gathered}
$$

where $a=0.4$ for $M=3$ and 0.5 otherwise. Obviously, the reference points for C2-DTLZ2 can be obtained by sampling
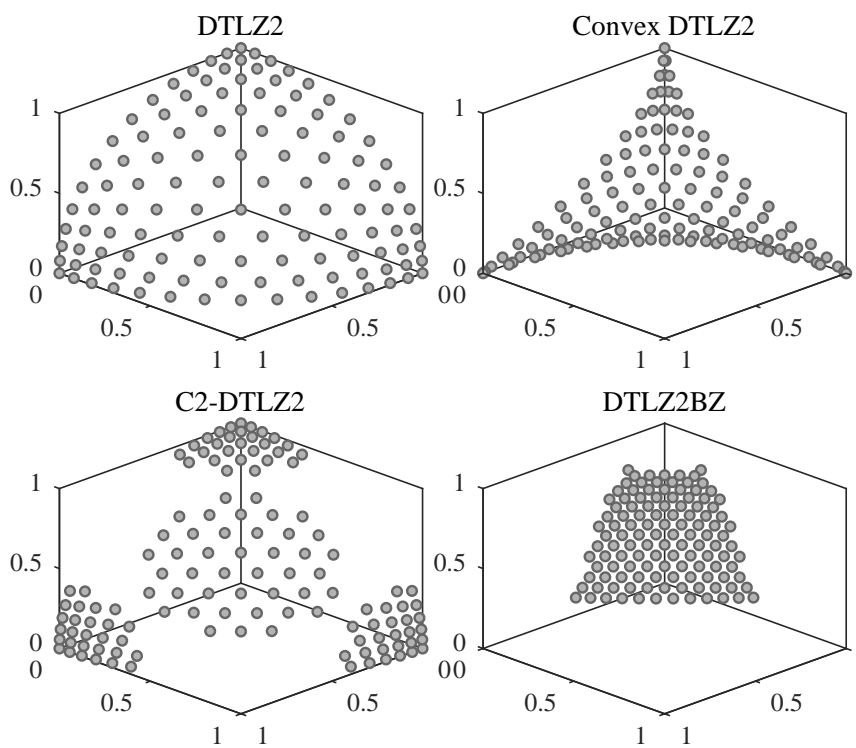

Fig. 7. Reference points sampled on 3-objective DTLZ2, convex DTLZ2, C2-DTLZ2, and DTLZ2BZ.

reference points on the PF of DTLZ2 and eliminating those do not satisfy the constraint, i.e.,

1) Generate a set of reference points $R$ for DTLZ2;

2) For each point $\left(r_{i 1}, r_{i 2}, \ldots, r_{i M}\right)$ in $R$, eliminate it if (16) is not satisfied, where $f_{1}, f_{2}, \ldots, f_{M}$ are replaced by $r_{i 1}, r_{i 2}, \ldots, r_{i M}$ in (16).

The PF of $M$-objective DTLZ2BZ is

$$
\left\{\begin{array}{l}
f_{1}=\cos \left(\theta_{1}\right) \ldots \cos \left(\theta_{M-2}\right) \cos \left(\theta_{M-1}\right) \\
f_{2}=\cos \left(\theta_{1}\right) \ldots \cos \left(\theta_{M-2}\right) \sin \left(\theta_{M-1}\right) \\
\ldots \\
f_{M}=\sin \left(\theta_{1}\right)
\end{array},\right.
$$

where $\theta_{j}=\frac{\pi}{2}\left(\frac{x_{j}}{2}+\frac{1}{4}\right)$ and $0 \leq x_{j} \leq 1$. Similar to C2-DTLZ2, the PF of DTLZ2BZ is also a part of the PF of DTLZ2, but the constraint cannot be represented by $f_{1}, f_{2}, \ldots, f_{M}$ like (16). Considering that the value of each $\theta_{j}$ is always between $\frac{1}{8} \pi$ and $\frac{3}{8} \pi$, for each reference point sampled on the PF of DTLZ2, we can calculate the values of $\theta_{1}, \theta_{2}, \ldots, \theta_{M-1}$ according to (17), and eliminate the reference point if any $\theta_{j}<\frac{1}{8} \pi$ or $\theta_{j}>\frac{3}{8} \pi$. As a result, the procedure of sampling reference points for DTLZ2BZ is

1) Generate a set of reference points $R$ for DTLZ2;

2) For each point $\left(r_{i 1}, r_{i 2}, \ldots, r_{i M}\right)$ in $R$, successively solve the values of $\theta_{M-1}, \theta_{M-2}, \ldots, \theta_{1}$ by $^{2}$

$$
\left\{\begin{array}{l}
\frac{\sin \left(\theta_{M-1}\right)}{\cos \left(\theta_{M-1}\right)}=\frac{r_{i 2}}{r_{i 1}} \\
\frac{\sin \left(\theta_{M-2}\right)}{\cos \left(\theta_{M-2}\right)}=\frac{r_{i 3}}{r_{i 2}} \sin \left(\theta_{M-1}\right) \\
\ldots \\
\frac{\sin \left(\theta_{1}\right)}{\cos \left(\theta_{1}\right)}=\frac{r_{i M}}{r_{i(M-1)}} \sin \left(\theta_{2}\right)
\end{array}\right.
$$

and eliminate the point if any $\theta_{j}<\frac{1}{8} \pi$ or $\theta_{j}>\frac{3}{8} \pi$.

\footnotetext{
${ }^{2}$ For simplicity, $r_{i j}=0$ can be replaced by a tiny value, e.g., 1e-6.
} 


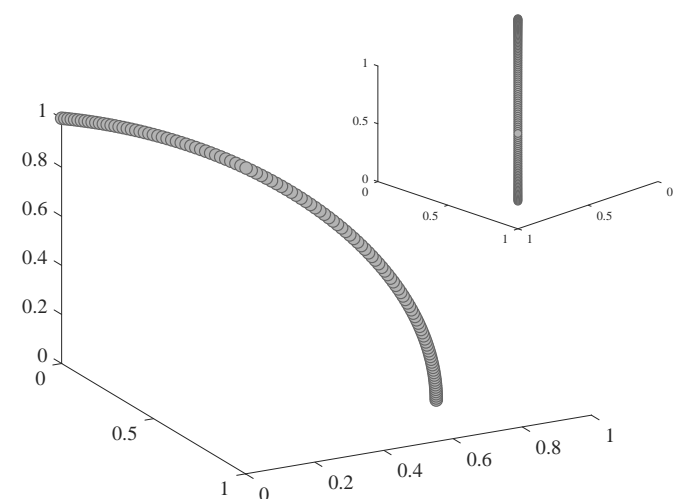

Fig. 8. 120 reference points sampled on 3-objective DTLZ5.

\section{Sampling Reference Points on Degenerate PF (DTLZ5)}

The PF of $M$-objective DTLZ5 [5] is

$$
\left\{\begin{array}{ll}
f_{j}=\left(\frac{1}{\sqrt{2}}\right)^{M-\max (j, 2)} \cos \left(\frac{\pi}{2} x\right), & j<M \\
f_{j}=\sin \left(\frac{\pi}{2} x\right), & j=M
\end{array},\right.
$$

where $0 \leq x \leq 1$. Since the PF of DTLZ5 is always a onedimensional curve independent of the number of objectives, the reference points for DTLZ5 can be obtained by sampling points on one-dimensional curve and extending them to the other objectives, i.e.,

1) Let $X$ be the set of a number of linearly equally spaced points between 0 and 1 ;

2) For each $x \in X$, calculate the objective values of the corresponding reference point by (19).

Fig. 8 shows 120 reference points sampled on the PF of 3-objective DTLZ5. It is worth to note that as pointed out in [6], [24], the PF of DTLZ5 with many objectives and decision variables is not totally a one-dimensional curve, which has an unknown non-degenerate part.

\section{Sampling Reference Points on Disconnected PF (DTLZ7)}

The PF of $M$-objective DTLZ7 [5] is:

$$
f_{M}=2 M-\sum_{j=1}^{M-1} f_{j}\left[1+\sin \left(3 \pi f_{j}\right)\right]
$$

where $0 \leq f_{j} \leq 1$ except for $f_{M}$. Note that DTLZ7 does not have a simplex-like PF; by contrast, the first $M-1$ objectives of the PF of DTLZ7 fill an $(M-1)$-dimensional unit hypercube, while the last objective is determined by (20). As a result, the procedure of sampling reference points for DTLZ7 is:

1) Replicate the vector $\left(\frac{0}{H}, \frac{1}{H}, \ldots, \frac{H}{H}\right)^{T}$ to produce an $(M-1)$-dimensional point set $X$, i.e., the number of points in $X$ is $(H+1)^{M-1,3}$

2) Let $R$ be the reference point set, for each $r_{i j} \in R$ and $x_{i j} \in X$,

$$
\left\{\begin{array}{ll}
r_{i j}=x_{i j}, & j<M \\
r_{i j}=2 M-\sum_{k=1}^{M-1} x_{i k}\left[1+\sin \left(3 \pi x_{i k}\right)\right], & j=M
\end{array} ;\right.
$$

\footnotetext{
${ }^{3}$ In order to obtain an arbitrary number of points in $X$, the good lattice point method or the Latin hypercube sampling can be adopted.
}

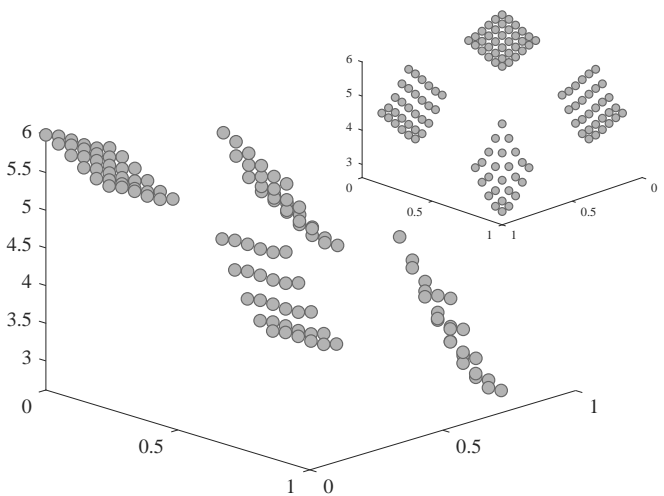

Fig. 9. 121 reference points sampled on 3-objective DTLZ7.

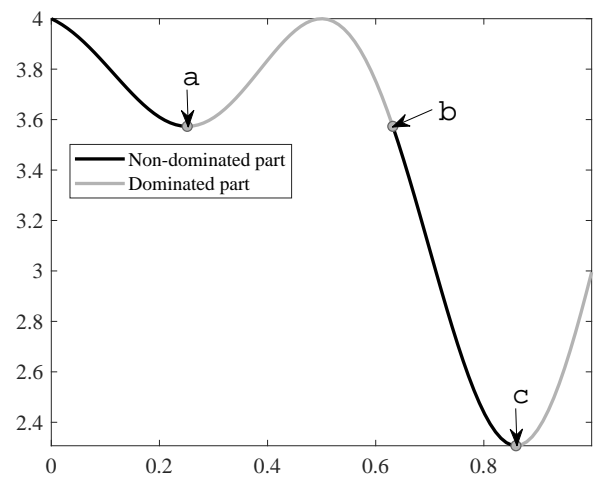

Fig. 10. The PF of 2-objective DTLZ7.

3) Delete the dominated points from $R$.

Fig. 9 plots 121 reference points sampled on the PF of 3objective DTLZ7. It is worth noting that the PF of DTLZ7 is disconnected, which means that there are many dominated points in $R$. For example, if $R$ contains 1024 points with 3 objectives, only 289 of which will be non-dominated. In fact, according to the PF of 2-objective DTLZ7 shown in Fig. 10, it is obvious that only the points between the origin and $a$ or $b$ and $c$ are non-dominated. According to (20), the first objective values of $a, b$ and $c$ are approximately $0.2514,0.6316$ and 0.8594 , respectively, hence the ratio of non-dominated points in $R$ for $M$-objective DTLZ7 is $\left(a_{1}-b_{1}+c_{1}\right)^{M-1} \approx 0.48^{M-1}$.

In order to increase the ratio of non-dominated points in $R$, we can map the elements in each column of $X$ from $[0,1]$ to $\left[0, a_{1}\right] \cup\left[b_{1}, c_{1}\right]$ before Step 2), so that all the points in $R$ are non-dominated and Step 3) can be eliminated.

\section{E. Sampling Reference Points on Highly Irregular PF (WFG2)}

The PF of WFG2 [6] is defined by

$$
\left\{\begin{array}{l}
f_{1}=2\left(1-\cos \left(\frac{\pi}{2} x_{1}\right)\right)\left(1-\cos \left(\frac{\pi}{2} x_{2}\right)\right) \ldots\left(1-\cos \left(\frac{\pi}{2} x_{M-1}\right)\right) \\
f_{2}=4\left(1-\cos \left(\frac{\pi}{2} x_{1}\right)\right)\left(1-\cos \left(\frac{\pi}{2} x_{2}\right)\right) \ldots\left(1-\sin \left(\frac{\pi}{2} x_{M-1}\right)\right) \\
\ldots \\
f_{M-1}=2(M-1)\left(1-\cos \left(\frac{\pi}{2} x_{1}\right)\right)\left(1-\sin \left(\frac{\pi}{2} x_{2}\right)\right) \\
f_{M}=2 M\left(1-x_{1} \cos ^{2}\left(5 \pi x_{1}\right)\right)
\end{array},\right.
$$

where $0 \leq x_{j} \leq 1$. Although the PF of WFG2 is simplelike, it cannot be defined as an equation represented by $f_{1}, f_{2}, \ldots, f_{M}$, hence the reference points cannot be mapped 


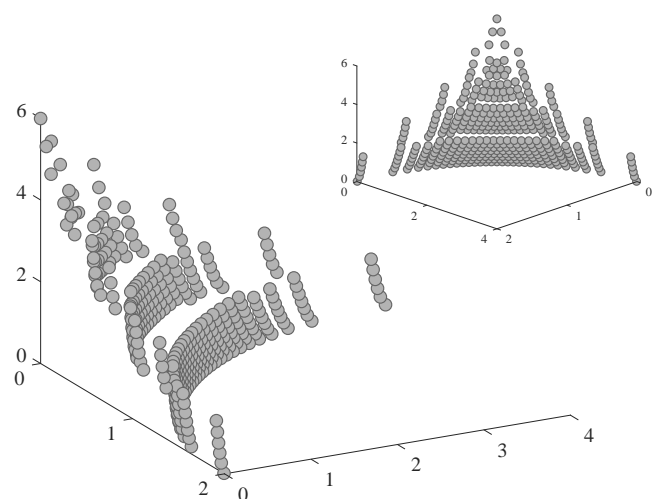

Fig. 11. 381 reference points sampled on 3-objective WFG2.

from unit simplex to the PF of WFG2 by the method used in DTLZ2 and convex DTLZ2. On the contrary, the mapping of reference points can be achieved by solving $x_{j}$, i.e.,

1) Uniformly sample a set of points $S$ on unit simplex;

2) For each point $\left(s_{i 1}, s_{i 2}, \ldots, s_{i M}\right)$ in $S$, successively solve the values of $x_{M-1}, x_{M-2}, \ldots, x_{1}$ by $^{4}$

$$
\left\{\begin{array}{l}
\frac{1-\sin \left(\frac{\pi}{2} x_{M-1}\right)}{1-\cos \left(\frac{\pi}{2} x_{M-1}\right)}=\frac{s_{i 2}}{s_{i 1}} \\
\frac{1-\sin \left(\frac{\pi}{2} x_{M-2}\right)}{1-\cos \left(\frac{\pi}{2} x_{M-2}\right)}=\frac{s_{i 3}}{s_{i 2}}\left(1-\sin \left(\frac{\pi}{2} x_{M-1}\right)\right) \\
\cdots \\
\frac{1-x_{1} \cos ^{2}\left(5 \pi x_{1}\right)}{1-\cos \left(\frac{\pi}{2} x_{1}\right)}=\frac{s_{i M}}{s_{i(M-1)}}\left(1-\sin \left(\frac{\pi}{2} x_{2}\right)\right)
\end{array},\right.
$$

and calculate the objective values of the corresponding reference point by (22).

3) Delete the dominated points from the reference point set.

Fig. 11 depicts 381 reference points sampled on the PF of 3-objective WFG2.

\section{F. Sampling Reference Points for MOPs Whose PFs are Unknown}

The above reference point sampling methods are applicable to most existing multi-objective test suites. However, so far the PFs of some MOPs are still unknown (e.g., WFG3 [6] and most combinatorial MOPs [25], [26], [27]), hence it is impossible to obtain reference points on their PFs. In order to calculate the performance metric values of the solutions obtained on these MOPs, an alternative sampling method is to combine all the non-dominated solutions obtained in multiple runs, and truncate the solutions by the truncation method used in SPEA2 [28].

For example, Fig. 13 plots 100 reference points sampled for 3-objective WFG3. It is worth to note that although the reference points generated by this method may not be on the true PF, it has been evidenced that they are suitable to reflect the difference in performance between MOEAs [14].

\section{EMPirical EVAluations}

To verify the effectiveness of the proposed reference point sampling methods in performance assessment, five selected

\footnotetext{
${ }^{4}$ According to the definition of Pareto front, in the case that $x_{1}$ has multiple solutions, the one minimizing $f_{M}$ is chosen.
}

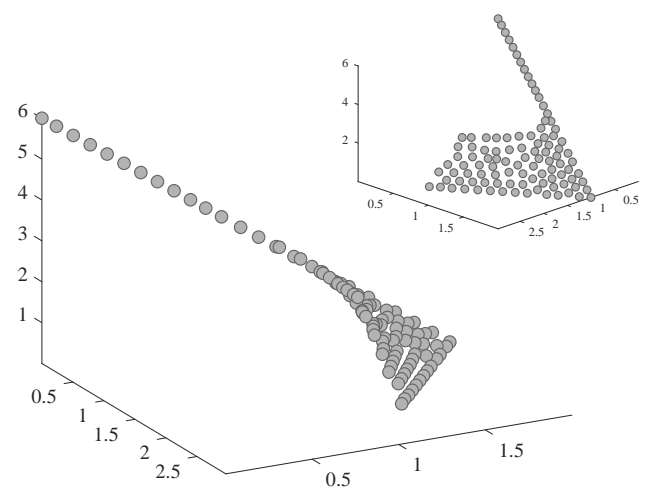

Fig. 13. 100 reference points sampled on 3-objective WFG3.

MOEAs (i.e, NSGA-II [29], RVEA [30], KnEA [31], NSGAIII [20], and AR-MOEA [32]) are tested on five popular MOPs (i.e., DTLZ1, DTLZ5, DTLZ7, C2-DTLZ2, and WFG2), where the results are analyzed by IGD based on the reference points generated by the proposed sampling methods.

For RVEA, the penalty parameter is set to 2 and the frequency of reference point adaption is set to 0.1. For KnEA, the rate of knee points is set to 0.5 . The population size of all the five MOEAs is set to 105 , and the maximum number of generations is set to 500. The simulated binary crossover [33] and polynomial mutation [34] are employed as the genetic operators, where the probabilities of crossover and mutation are set to 1 and $1 / D$ ( $D$ denotes the number of decision variables), respectively, and the distribution index is set to 20 . All the tests are run for 30 times independently.

Table I lists the IGD values obtained by the five MOEAs, where roughly 10000 reference points are generated on each $\mathrm{PF}$ by the proposed sampling methods. It can be seen from the statistical results that RVEA, NSGA-III, and AR-MOEA have the best IGD values on DTLZ1 and WFG2, while AR-MOEA has significantly better IGD values than the others on DTLZ5, DTLZ7, and C2-DTLZ2. Fig. 12 plots the results with the median IGD among 30 runs, from which it is obvious that the IGD results based on the proposed reference point sampling methods are consistent with the visual conclusion.

For comparison, Table II presents the IGD values based on randomly sampled reference points, where some of the results are clearly counter-intuitive. For example, according to Table II, NSGA-III has better IGD value than KnEA on DTLZ5; however, the solutions obtained by NSGA-III distribute obviously less widely than those obtained by KnEA as shown in Fig. 12. Similar inconsistencies can also be found between the IGD values of NSGA-II and RVEA on C2-DTLZ2 and WFG2. Therefore, the randomly sampled reference points are unsuitable for the calculation of performance metrics.

\section{CONCLUSIONS}

Sampling uniformly distributed reference points on PFs is an important but neglected issue. In order to fill this gap, this paper first details three popular methods for sampling reference points on unit simplex, then introduces several methods for sampling reference points on different types of 

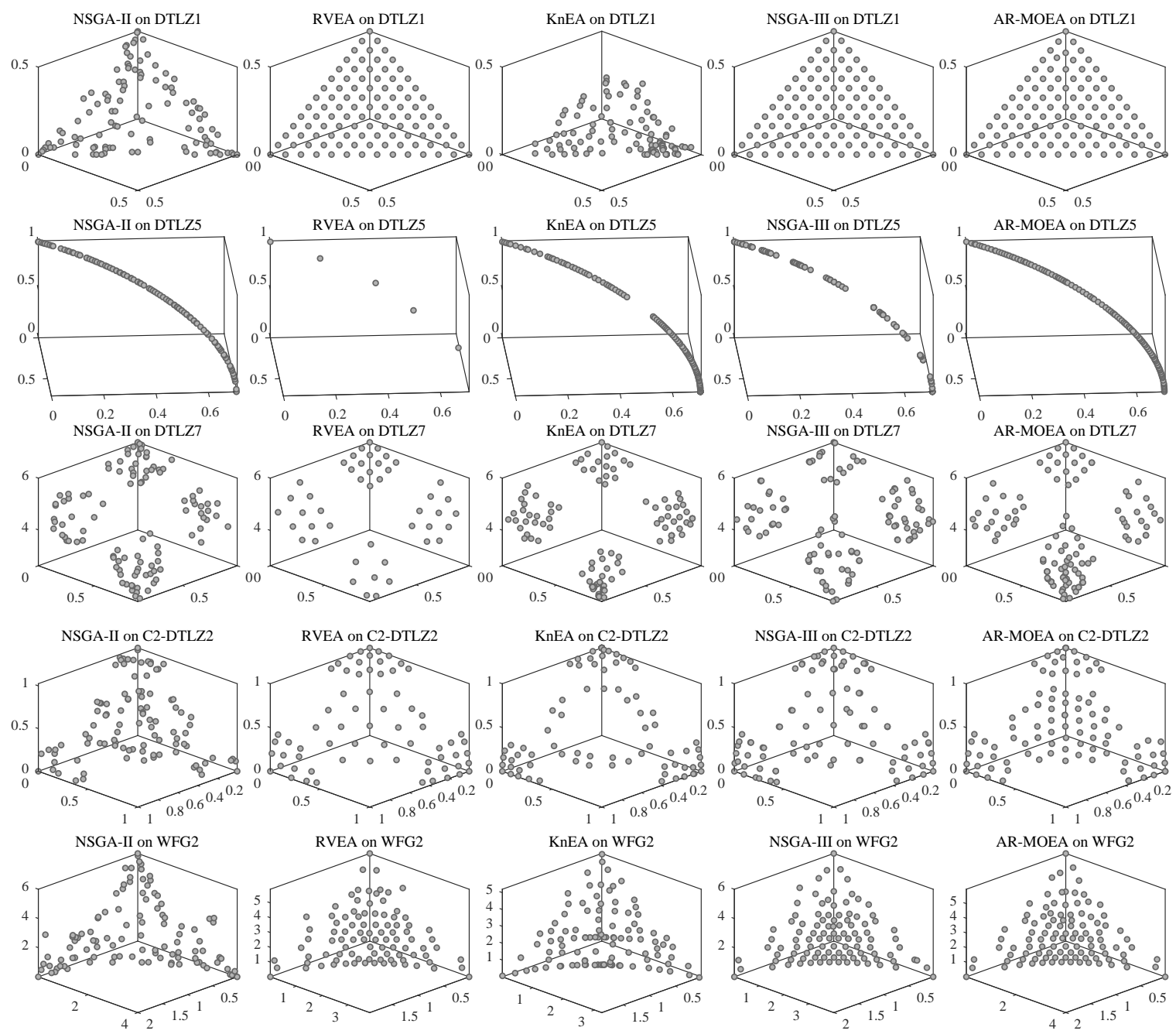

Fig. 12. The non-dominated solution sets with the median IGD among 30 runs obtained by NSGA-II, RVEA, KnEA, NSGA-III, and AR-MOEA on 3-objective DTLZ1, DTLZ5, DTLZ7, C2-DTLZ2, and WFG2.

TABLE I

IGD Values Based on Uniformly Sampled Reference Points Obtained by NSGA-II, RVEA, KnEA, NSGA-III, AND AR-MOEA on 3-OBJECTIVE DTLZ1, DTLZ5, DTLZ7, C2-DTLZ2, AND WFG2.

\begin{tabular}{cccccc}
\hline Problem & NSGA-II & RVEA & KnEA & NSGA-III & AR-MOEA \\
\hline DTLZ1 & $2.6500 \mathrm{e}-2(1.11 \mathrm{e}-3)$ & $1.9012 \mathrm{e}-2(3.01 \mathrm{e}-5)$ & $4.5373 \mathrm{e}-2(1.79 \mathrm{e}-2)$ & $1.9057 \mathrm{e}-2(1.09 \mathrm{e}-4)$ & $1.9038 \mathrm{e}-2(5.57 \mathrm{e}-5)$ \\
DTLZ5 & $5.5807 \mathrm{e}-3(3.24 \mathrm{e}-4)$ & $6.9232 \mathrm{e}-2(1.14 \mathrm{e}-2)$ & $1.7279 \mathrm{e}-2(1.19 \mathrm{e}-2)$ & $1.8106 \mathrm{e}-2(2.37 \mathrm{e}-3)$ & $4.2931 \mathrm{e}-3(7.23 \mathrm{e}-5)$ \\
DTLZ7 & $7.4402 \mathrm{e}-2(3.86 \mathrm{e}-3)$ & $1.0457 \mathrm{e}-1(4.34 \mathrm{e}-3)$ & $6.7288 \mathrm{e}-2(4.55 \mathrm{e}-3)$ & $7.0937 \mathrm{e}-2(2.14 \mathrm{e}-3)$ & $6.2788 \mathrm{e}-2(1.09 \mathrm{e}-3)$ \\
C2-DTLZ2 & $5.5011 \mathrm{e}-2(1.88 \mathrm{e}-3)$ & $5.2301 \mathrm{e}-2(1.04 \mathrm{e}-3)$ & $7.9084 \mathrm{e}-2(4.06 \mathrm{e}-2)$ & $4.8558 \mathrm{e}-2(5.98 \mathrm{e}-4)$ & $4.4312 \mathrm{e}-2(5.94 \mathrm{e}-4)$ \\
WFG2 & $2.1386 \mathrm{e}-1(9.25 \mathrm{e}-3)$ & $1.4977 \mathrm{e}-1(1.47 \mathrm{e}-3)$ & $1.8477 \mathrm{e}-1(1.10 \mathrm{e}-2)$ & $1.6366 \mathrm{e}-1(3.35 \mathrm{e}-3)$ & $1.4923 \mathrm{e}-1(9.86 \mathrm{e}-4)$ \\
\hline
\end{tabular}

TABLE II

IGD Values Based on Randomly Sampled Reference Points ObTained by NSGA-II, RVEA, KNEA, NSGA-III, AND AR-MOEA on 3-ObJective DTLZ1, DTLZ5, DTLZ7, C2-DTLZ2, AND WFG2. The RESUlts InCONSISTENT With Fig. 12 In EACH Row ARE Framed.

\begin{tabular}{|c|c|c|c|c|c|}
\hline Problem & NSGA-II & RVEA & KnEA & NSGA-III & AR-MOEA \\
\hline DTLZ1 & $2.4647 \mathrm{e}-2(1.07 \mathrm{e}-3)$ & $1.9008 \mathrm{e}-2(3.25 \mathrm{e}-5)$ & $5.9984 \mathrm{e}-2(1.98 \mathrm{e}-2)$ & $1.9063 \mathrm{e}-2(1.32 \mathrm{e}-4)$ & $1.9035 \mathrm{e}-2(6.84 \mathrm{e}-5)$ \\
\hline DTLZ5 & $5.4692 \mathrm{e}-3(2.63 \mathrm{e}-4)$ & $6.8352 \mathrm{e}-2(6.75 \mathrm{e}-3)$ & $2.0950 \mathrm{e}-2(1.52 \mathrm{e}-2)$ & $1.9120 \mathrm{e}-2(2.46 \mathrm{e}-3)$ & $4.2935 \mathrm{e}-3(5.52 \mathrm{e}-5)$ \\
\hline DTLZ7 & $7.3551 \mathrm{e}-2(3.13 \mathrm{e}-3)$ & $1.0276 \mathrm{e}-1(4.55 \mathrm{e}-3)$ & $6.7255 \mathrm{e}-2(4.06 \mathrm{e}-3)$ & $7.1555 \mathrm{e}-2(2.27 \mathrm{e}-3)$ & $6.2552 \mathrm{e}-2(9.64 \mathrm{e}-4)$ \\
\hline C2-DTLZ2 & $5.0972 \mathrm{e}-2(1.41 \mathrm{e}-3)$ & $5.1628 \mathrm{e}-2(3.95 \mathrm{e}-4)$ & $8.5015 \mathrm{e}-2(4.71 \mathrm{e}-2)$ & $4.9519 \mathrm{e}-2(8.12 \mathrm{e}-4)$ & $4.3762 \mathrm{e}-2(5.24 \mathrm{e}-4)$ \\
\hline WFG2 & $1.6601 \mathrm{e}-1(1.41 \mathrm{e}-2)$ & $1.8192 \mathrm{e}-1(8.22 \mathrm{e}-3)$ & $2.6463 \mathrm{e}-1(8.32 \mathrm{e}-2)$ & $1.9448 \mathrm{e}-1(6.62 \mathrm{e}-3)$ & $1.9260 \mathrm{e}-1(8.57 \mathrm{e}-3)$ \\
\hline
\end{tabular}


PFs. Experimental results demonstrate that the reference points generated by the proposed sampling methods can evaluate the performance of MOEAs accurately.

As mentioned before, the proposed reference point sampling methods can cover most of the existing multi-objective test suites. This is because existing benchmark MOPs contain only a few types of PFs, where most of these PFs are very similar to those presented in this paper. Therefore, in order to better evaluate the comprehensive performance of MOEAs, it is desirable to design new MOPs with more complex PFs to pose stiffer challenges to MOEAs in the future.

\section{ACKNOWLEDGMENT}

This work was supported in part by the National Natural Science Foundation of China (Grant No. 61672033, 61502004, and 61502001). The work of Y. Jin was supported in part by the U.K. EPSRC Under Grant EP/M017869/1.

\section{REFERENCES}

[1] J. D. Schaffer, "Multiple objective optimization with vector evaluated genetic algorithms," in Proceedings of the 1st International Conference on Genetic Algorithms, 1985, pp. 93-100.

[2] A. Zhou, B.-Y. Qu, H. Li, S.-Z. Zhao, P. N. Suganthan, and Q. Zhang, "Multiobjective evolutionary algorithms: A survey of the state of the art," Swarm and Evolutionary Computation, vol. 1, no. 1, pp. 32-49, 2011.

[3] B. Li, J. Li, K. Tang, and X. Yao, "Many-objective evolutionary algorithms: A survey," ACM Computing Surveys, vol. 48, no. 1, p. 13, 2015.

[4] E. Zitzler, K. Deb, and L. Thiele, "Comparison of multiobjective evolutionary algorithms: Empirical results," Evolutionary Computation, vol. 8, no. 2, pp. 173-195, 2000

[5] K. Deb, L. Thiele, M. Laumanns, and E. Zitzler, "Scalable test problems for evolutionary multiobjective optimization," in Evolutionary Multiobjective Optimization. Theoretical Advances and Applications, 2005, pp. 105-145.

[6] L. B. S. Huband, P. Hingston and L. While, "A review of multiobjective test problems and a scalable test problem toolkit," IEEE Transactions on Evolutionary Computation, vol. 10, no. 5, pp. 477-506, 2006.

[7] Q. Zhang, A. Zhou, S. Zhao, P. N. Suganthan, W. Liu, and S. Tiwari, "Multiobjective optimization test instances for the CEC 2009 special session and competition," University of Essex, Colchester, UK and Nanyang technological University, Tech. Rep. CES-487, Tech. Rep., 2008.

[8] R. Cheng, Y. Jin, M. Olhofer, and B. Sendhoff, "Test problems for largescale multiobjective and many-objective optimization," IEEE Transactions on Cybernetics, 2016, in press.

[9] R. Cheng, M. Li, Y. Tian, X. Zhang, S. Yang, Y. Jin, and X. Yao, "A benchmark test suite for evolutionary many-objective optimization," Complex \& Intelligent Systems, vol. 3, no. 1, pp. 67-81, 2017.

[10] D. A. V. Veldhuizen and G. B. Lamont, "Multiobjective evolutionary algorithm research: A history and analysis," Department of Electrical and Computer Engineering. Graduate School of Engineering, Air Force Inst Technol, Wright Patterson, Tech. Rep. TR-98-03, Tech. Rep., 1998.

[11] A. Zhou, Y. Jin, Q. Zhang, B. Sendhoff, and E. Tsang, "Combining model-based and genetics-based offspring generation for multi-objective optimization using a convergence criterion," in Proceedings of the 2006 IEEE Congress on Evolutionary Computation, 2006, pp. 892-899.

[12] L. While, P. Hingston, L. Barone, and S. Huband, "A faster algorithm for calculating hypervolume," IEEE Transactions on Evolutionary Computation, vol. 10, no. 1, pp. 29-38, 2006.

[13] K. Li, K. Deb, Q. Zhang, and S. Kwong, "Combining dominance and decomposition in evolutionary many-objective optimization," IEEE Transactions on Evolutionary Computation, vol. 19, no. 5, pp. 694-716, 2015.

[14] H. Ishibuchi, H. Masuda, and Y. Nojima, "Pareto fronts of manyobjective degenerate test problems," IEEE Transactions on Evolutionary Computation, vol. 20, no. 5, pp. 807-813, 2016.
[15] Y. Tian, R. Cheng, X. Zhang, and Y. Jin, "PlatEMO: A MATLAB platform for evolutionary multi-objective optimization," IEEE Cотриtational Intelligence Magazine, vol. 12, no. 4, pp. 73-87, 2017.

[16] C. He, L. Pan, H. Xu, Y. Tian, and X. Zhang, "An improved reference point sampling method on Pareto optimal front," in Proceedings of the 2016 IEEE Congress on Evolutionary Computation, 2016, pp. 52305237.

[17] S. Jiang and S. Yang, "A strength Pareto evolutionary algorithm based on reference direction for multi-objective and many-objective optimization," IEEE Transactions on Evolutionary Computation, vol. 21, no. 3, pp. 329-346, 2017.

[18] I. Das and J. E. Dennis, "Normal-boundary intersection: A new method for generating the Pareto surface in nonlinear multicriteria optimization problems," SIAM Journal on Optimization, vol. 8, no. 3, pp. 631-657, 1998.

[19] Q. Zhang and H. Li, "MOEA/D: A multi-objective evolutionary algorithm based on decomposition," IEEE Transactions on Evolutionary Computation, vol. 11, no. 6, pp. 712-731, 2007.

[20] K. Deb and H. Jain, "An evolutionary many-objective optimization algorithm using reference-point based non-dominated sorting approach, part I: Solving problems with box constraints," IEEE Transactions on Evolutionary Computation, vol. 18, no. 4, pp. 577-601, 2014.

[21] K. Fang and C. Ma, Orthogonal and uniform experimental design. Science and Technology Press, Beijing, 2001.

[22] H. Jain and K. Deb, "An evolutionary many-objective optimization algorithm using reference-point based nondominated sorting approach, part II: Handling constraints and extending to an adaptive approach," IEEE Transactions on Evolutionary Computation, vol. 18, no. 4, pp. 602-622, 2014

[23] D. Brockhoff and E. Zitzler, "Objective reduction in evolutionary multiobjective optimization: Theory and applications," Evolutionary Computation, vol. 17, no. 2, pp. 135-166, 2009.

[24] D. K. Saxena, J. A. Duro, A. Tiwari, K. Deb, and Q. Zhang, "Objective reduction in many-objective optimization: Linear and nonlinear algorithms," IEEE Transactions on Evolutionary Computation, vol. 17, no. 1 , pp. 77-99, 2013.

[25] E. Zitzler and L. Thiele, "Multiobjective evolutionary algorithms: A comparative case study and the strength Pareto approach," IEEE transactions on Evolutionary Computation, vol. 3, no. 4, pp. 257-271, 1999

[26] D. B. Fogel, "An evolutionary approach to the traveling salesman problem," Biological Cybernetics, vol. 60, no. 2, pp. 139-144, 1988.

[27] Y. Zhang, M. Harman, and S. A. Mansouri, "The multi-objective next release problem," in Proceedings of the 9th Annual Conference on Genetic and Evolutionary Computation, 2007, pp. 1129-1137.

[28] E. Zitzler, M. Laumanns, and L. Thiele, "SPEA2: Improving the strength Pareto evolutionary algorithm for multiobjective optimization," in Proceedings of the Fifth Conference on Evolutionary Methods for Design, Optimization and Control with Applications to Industrial Problems, 2001, pp. 95-100.

[29] K. Deb, A. Pratap, S. Agarwal, and T. Meyarivan, "A fast and elitist multi-objective genetic algorithm: NSGA-II," IEEE Transactions on Evolutionary Computation, vol. 6, no. 2, pp. 182-197, 2002.

[30] R. Cheng, Y. Jin, M. Olhofer, and B. Sendhoff, "A reference vector guided evolutionary algorithm for many-objective optimization," IEEE Transactions on Evolutionary Computation, vol. 20, no. 5, pp. 773-791, 2016.

[31] X. Zhang, Y. Tian, and Y. Jin, "A knee point driven evolutionary algorithm for many-objective optimization," IEEE Transactions on Evolutionary Computation, vol. 19, no. 6, pp. 761-776, 2015.

[32] Y. Tian, R. Cheng, X. Zhang, F. Cheng, and Y. Jin, "An indicator based multi-objective evolutionary algorithm with reference point adaptation for better versatility," IEEE Transactions on Evolutionary Computation, 2017 , in press.

[33] K. Deb, Multi-Objective Optimization Using Evolutionary Algorithms. New York: Wiley, 2001.

[34] K. Deb and M. Goyal, "A combined genetic adaptive search (GeneAS) for engineering design," Computer Science and Informatics, vol. 26, no. 4, pp. 30-45, 1996 . 